\title{
CtDNA-identifying cancer before it is clinically detectable
}

Diffuse large B-cell lymphoma (DLBCL) is curable in patients treated with first-line therapy; however, $40 \%$ of patients are not cured and have disease recurrence. CT imaging is used to monitor patients who are likely to relapse following treatment, but is not optimal, exposes patients to radiation and is invasive. Next-generation sequencing (NGS) can detect and quantify circulating tumour DNA (ctDNA) noninvasively. Using NGS, Wyndham Wilson and coauthors assessed ctDNA clonal immunoglobulin rearranged sequences in the serum of patients with DLBCL and identified those at risk of recurrence before clinical evidence of disease.

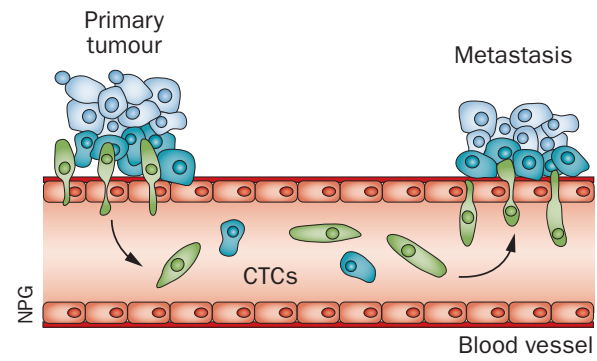

"We hypothesized that ctDNA in the blood may be used to identify patients at risk of treatment failure, and that it would be more sensitive than CT scans," explains Wilson. He continues, "we were able to conduct this study because we stored pretreatment tumour samples and serum samples from every clinical visit from our studies in patients with untreated DLBCL for the past 20 years.”

The researchers showed that ctDNA monitoring in patients who achieved remission had a high positive and negative predictive value for recurrence. Importantly, ctDNA identified patients with recurrence at a median of 3.5 months before evidence of disease on CT scans. They also examined the association of interim ctDNA after patients received two cycles of treatment to determine if patients at higher risk of treatment failure could be identified. Surveillance monitoring of ctDNA was performed in 107 patients who achieved complete remission. In patients who developed detectable ctDNA the risk of disease progression was over 200 times greater than for patients with undetectable ctDNA. Moreover, patients who never cleared ctDNA had a much shorter time to disease progression than those with transient clearance.

"These results suggest that ctDNA monitoring is a very effective method of disease assessment and seems to be more sensitive than CT imaging. Furthermore, it can be used to quantitatively assess tumour load and kinetics, which may be important for identifying patients who may need less treatment as well as those who require alternative treatments." This technology could be useful for clinical decisions regarding maintenance treatment, and in the early assessment of response to targeted therapies ultimately to enhance personalized treatment.

\section{Lisa Hutchinson}

Original article Roschewski, M. et al. Circulating tumour DNA and CT monitoring in patients with untreated diffuse large B-cell lymphoma: a correlative biomarker study. Lancet Oncol. doi:10.1016/S1470-2045(15)70106-3 